\title{
Galois Field-based Approach for Rotation and Scale Invariant Texture Classification
}

\author{
Shivashankar S. \\ Dept of Computer Science, Karnatak University, Dharwad, 580003, India \\ Email: shivashankars@kud.ac.in \\ Medha Kudari \\ Dept of Computer Science, Karnatak University, Dharwad, 580003, India \\ Email: medha.k27@gmail.com \\ Prakash S. Hiremath \\ Dept of Computer Science (MCA), KLE Technological University, BVBCET, Hubballi, 580031, India \\ Email: hiremathps53@yahoo.com
}

Received: 04 April 2018; Accepted: 14 June 2018; Published: 08 September 2018

\begin{abstract}
In this paper, a novel Galois Field-based approach is proposed for rotation and scale invariant texture classification. The commutative and associative properties of Galois Field addition operator are useful for accomplishing the rotation and scale invariance of texture representation. Firstly, the Galois field operator is constructed, which is applied to the input textural image. The normalized cumulative histogram is constructed for Galois Field operated image. The bin values of the histogram are considered as rotation and scale invariant texture features. The classification is performed using the K-Nearest Neighbour classifier. The experimental results of the proposed method are compared with that of Rotation Invariant Local Binary Pattern (RILBP) and Log-Polar transform methods. These results obtained using the proposed method are encouraging and show the possibility of classifying texture successfully irrespective of its rotation and scale.
\end{abstract}

Index Terms-Galois Field representation of texture image, Feature histogram computation, Rotation and scale invariance, Texture classification.

\section{INTRODUCTION}

A texture is a prime characteristic of surface properties of an object that distinguish it from other objects in an image. Texture analysis is very important in image processing and computer vision. It involves representing the characteristics of image regions in a form that can identify the type of image texture from a finite set of texture classes. Remote sensing analysis, medical image interpretation, pattern recognition and content-based image retrieval are some of the image-based applications, where texture analysis plays a fundamental and important role $[1,2]$.
Texture representations can be classified into five categories in terms of the feature types employed, namely, statistical [3], structural [4], geometrical [5], model based [6] and signal processing features [7]. The majority of existing texture analysis methods make assumption that texture images are acquired from the same viewpoint (i.e. the same scale and orientation). In image analysis applications, it is difficult to ensure that a captured texture image has the same viewpoint as the training images. Despite decades of research efforts, texture description remains a challenging problem, because of many variations that can affect texture patterns like rotation, scale, illumination and so on. Hence, invariant texture description is highly desirable from both theoretical and practical perspectives. Thus, many researchers address rotation and scale invariance issues in their work on texture analysis [8].

\section{RELATED WORK}

Rotational invariance is achieved using a model based approach in [9] and signal processing approach in [5]. Other popular methods for rotation invariance texture classification include statistical approaches like Local Binary Patterns [10], wavelets [11], Gabor filters [12], Radon transform and Discrete Cosine Transform [13]. Recently rotation invariance has been obtained by a combination of extremum filtering, feature transform, scalar quantization and cross-scale joint coding for representing histograms in [14].

Scale invariance has been obtained by designing a histogram using Local Binary Pattern and fractal dimension image weights in [15]. Other scale invariant texture description methods include fractals [16], random projection [17], Harris and Laplacian key points [18] and 


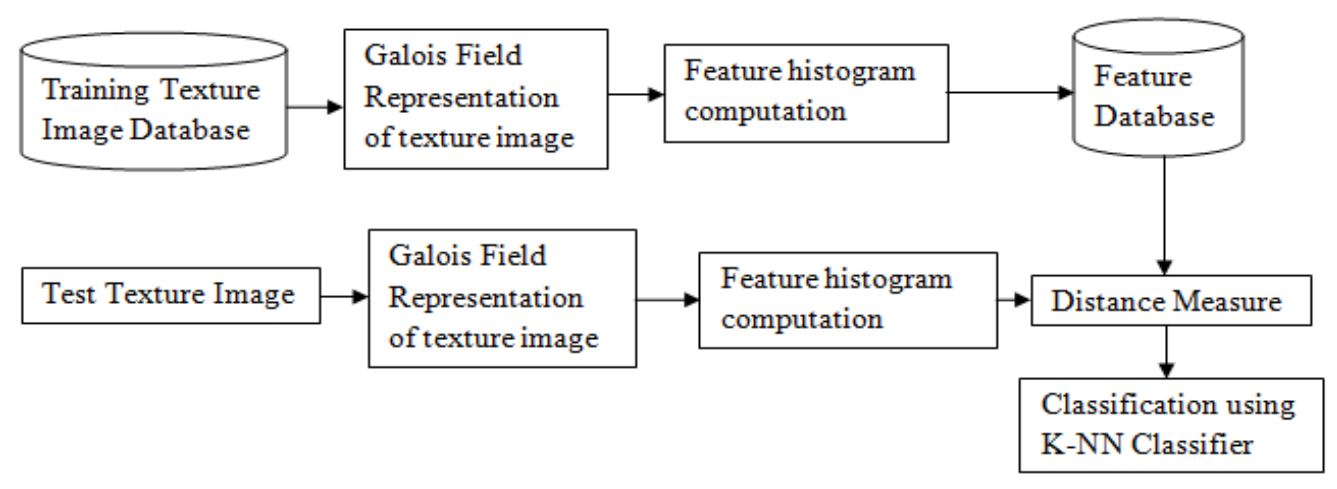

Fig.1. Flow design of the proposed method

pyramid histograms [19]. In [20], scale invariant classification of texture images in frequency domain has been achieved, whereas statistical approach is used in [21] and geometric approach is discussed in [19].

Despite its importance, work on simultaneous scale and rotation invariant texture representation is limited. The major existing approaches include psycho-physical transformation, multi-resolution simultaneous autoregressive (MRSAR) model [22], log-polar wavelet signatures [23], multichannel Gabor filtering [12, 24] and the Wold model [25] for invariant texture analysis.

Galois Fields has gained widespread applications in modern computer systems such as computer networks, satellite links or storage systems. These systems use finite field arithmetic for error correction or for cryptographic algorithms. Reed, Truong, Kwoh and Hall [26] proposed a Galois Field transform method for twodimensional filtering which is faster than the conventional Fast Fourier Transform algorithm.

In the present work, Galois Field-based approach in [27] is extended for rotation and scale invariant texture classification. Firstly, the Galois field operator is constructed, which is applied to the input textural image. The bin values of the normalized cumulative histogram constructed for Galois Field operated image are considered as texture features. These features are inherently rotation and scale invariant due to the commutative and associative properties of Galois Field addition operator. These features are used for texture image classification using K-Nearest Neighbour classifier. The experimentation is performed on four benchmark texture datasets, namely, Brodatz, Mondial Marmi, Outex and Vectorial. The results are compared with that of Rotation Invariant Local Binary Pattern (RILBP) [10] and Log-Polar transform methods [23]. It is observed that Galois Field based approach achieves better classification accuracy when compared to other two methods.

The rest of the paper is organized as follows: The texture representation in Galois Field is described in Section III. In Section IV, the feature extraction and classification process is discussed. Experimental results and discussion is presented in Section V. Finally, Section VI gives conclusion.

\section{TeXture REPRESENTATION IN GALOIS FIELD (GF)}

The flow design of the proposed method is shown in Fig. 1. Firstly, the Galois field operator is constructed, which is applied to the input textural image. A Galois Field (GF) is a finite field that contains a finite number of elements, called its order. Galois fields are given by the integers modulo a prime. i.e. for any prime integer $\mathrm{p}$ and any integer $n$ greater than or equal to 1 , there is a unique field with $\mathrm{p}^{\mathrm{n}}$ elements in it, denoted by $\operatorname{GF}\left(\mathrm{p}^{\mathrm{n}}\right)$.

Consider a gray scale image I with intensity values in the range 0 to 255 which are represented in 8-bit binary form in the memory. Each pixel intensity value in its binary form can be considered as an element in a Finite Field of $2^{8}$, i.e., Galois Field $\operatorname{GF}\left(2^{8}\right)$. We define a Galois Field operator as described below: Consider a 3 x 3 neighbourhood of a pixel $\mathrm{I}_{\mathrm{i}, \mathrm{j}}$ in an image I. In $\mathrm{GF}\left(2^{8}\right)$, perform mod 2 addition (denoted by $\oplus$ ) of eight neighbouring elements along with center pixel $I_{i, j}$ resulting in a 8-bit binary value $\mathrm{I}_{\mathrm{i}, \mathrm{j}}$. The addition of all the nine elements in GF yields the same value irrespective of the order in which these elements are added. This GF operator is applied to all the pixels in the image I, which results in a transformed image I'.

For numerical illustration, consider the $3 \times x$ neighbourhood of $I_{i, j}$ as shown in Fig. 2.

\begin{tabular}{|l|l|l|}
\hline 01111010 & 01100111 & 01011100 \\
\hline 10100110 & 01111001 & 01011101 \\
\hline 11010101 & 10100000 & 01101011 \\
\hline
\end{tabular}

(a)

\begin{tabular}{|c|c|c|}
\hline$I_{i-1, j-1}$ & $I_{i-1, j}$ & $I_{i-1, j+1}$ \\
\hline$I_{i, j-1}$ & $I_{i, j}$ & $I_{i, j+1}$ \\
\hline$I_{i+1, j-1}$ & $I_{i+1, j}$ & $I_{i+1, j+1}$ \\
\hline
\end{tabular}

(b)

Fig.2 (a). 8-bit binary form of $3 \times 3$ portion of an image I, (b). 8-nearest neighbourhood of $\mathrm{I}_{\mathrm{i},}$ 
For example, the result $\mathrm{I}_{\mathrm{i}, \mathrm{j}}$, that would be obtained when Galois Field operator is performed on $3 \times 3$ neighbourhood of $I_{i, j}$ Fig. 2(b), is computed as:

$$
\begin{array}{r}
\mathrm{I}_{\mathrm{i}, \mathrm{j}}{ }=\left(\left(\left(\left(\left(\left(\left(\left(\mathrm{I}_{\mathrm{i}, \mathrm{j}} \oplus \mathrm{I}_{\mathrm{i}-1, \mathrm{j}}\right) \oplus \mathrm{I}_{\mathrm{i}-1, \mathrm{j}-1}\right) \oplus \mathrm{I}_{\mathrm{i}, \mathrm{j}-1}\right) \oplus \mathrm{I}_{\mathrm{i}+1, \mathrm{j}-1}\right)\right.\right.\right.\right. \\
\left.\left.\left.\left.\oplus \mathrm{I}_{\mathrm{i}+1, \mathrm{j}}\right) \oplus \mathrm{I}_{\mathrm{i}+1, \mathrm{j}+1}\right) \oplus \mathrm{I}_{\mathrm{i}, \mathrm{j}+1}\right) \oplus \mathrm{I}_{\mathrm{i}-1, \mathrm{j}+1}\right)
\end{array}
$$

The value obtained for portion of an image I shown in Fig. 2(a) is

$$
\mathrm{I}_{\mathrm{i}, \mathrm{j}}^{\prime}=(11011101)_{2}=(221)_{10}
$$

\section{A FEATURE EXTRACTION}

The histogram is constructed for the GF operated image. The histogram is represented as a discrete function

$$
\mathrm{h}\left(\mathrm{r}_{\mathrm{k}}\right)=\mathrm{n}_{\mathrm{k}}
$$

where $r_{k}$ is the $k^{\text {th }}$ value and $n_{k}$ is the number of $r_{k}$ values in the GF operated image.

The cumulative histogram is computed as

$$
\mathrm{C}_{\mathrm{k}}=\sum_{\mathrm{j}=0}^{\mathrm{k}} \mathrm{h}\left(\mathrm{r}_{\mathrm{j}}\right)
$$

where $\mathrm{k}=0,1,2, \ldots, 255$.

The normalized cumulative histogram is given by

$$
\mathrm{NC}_{\mathrm{k}}=\frac{\mathrm{C}_{\mathrm{k}}}{\|\mathrm{C}\|}
$$

Where

$$
\|C\|=\sqrt{c_{0}^{2}+c_{1}^{2}+\ldots .+c_{k}^{2}+\ldots .+c_{255}^{2}}
$$

The bin values of the normalized cumulative histograms $\mathrm{NC}_{\mathrm{k}}$ are used for feature construction. The resultant features are considered as the rotation and scale invariant texture features.

\section{B. Classification}

The process of texture classification is to identify the type of unknown texture from a finite set of known texture classes. The classification approach comprises of two stages, namely, training and testing. The training stage consists of extracting the features from the training samples using the proposed method and is stored in the database for testing. The testing stage consists of extracting similar features from the test sample. The test sample is classified using the K-Nearest Neighbour classifier based on the similarity measure. The similarity between the test sample and the trained sample is measured by using the Euclidean distance metric [28] defined as:

$$
d(p, q)=\sqrt{\sum_{i}\left(p_{i}-q_{i}\right)^{2}}
$$

where $p_{i}, q_{i}$ are the $i^{\text {th }}$ feature of test sample and training sample, respectively.

\section{EXPERIMENTAL RESULTS AND DISCUSSION}

The experimentation is performed to evaluate the effectiveness of the proposed feature representation method in rotation and scale invariant texture classification.

\section{A. Data Set}

The four benchmark texture datasets, namely, Brodatz [29], Mondial Marmi [30], Outex [31] and Vectorial [32] of planar images, which were considered by Bianconi and Fernandez [7], are used for experimentation.

\section{1) Brodatz}

The dataset has the following thirteen texture classes, namely, D112, D94, D92, D84, D68, D38, D29, D24, D19, D16, D15, D12 and D9. Each image is subdivided into 16 non-overlapping subimages that are of size $205 \mathrm{x}$ 205 pixels. This results in 16 samples for each class. The dataset contains the images rotated in steps of $10^{\circ}$ ranging from $0^{\circ}$ to $90^{\circ}$ [29]. The sample images of the texture class D84 for different rotation angles $0^{\circ}$ to $90^{\circ}$ in steps of $10^{\circ}$ are shown in Fig. 3 .

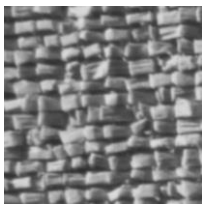

$0^{\circ}$

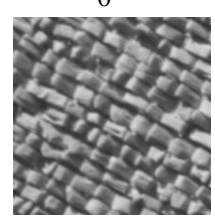

$30^{\circ}$

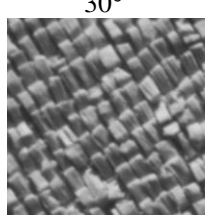

$60^{\circ}$

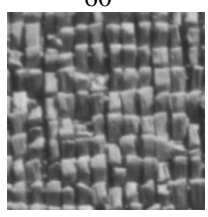

$90^{\circ}$

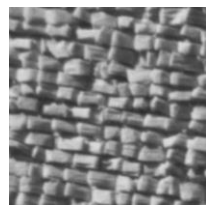

$10^{\circ}$

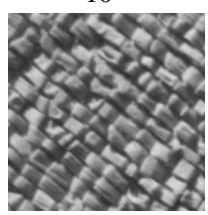

$40^{\circ}$

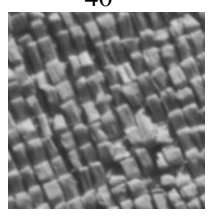

$70^{\circ}$

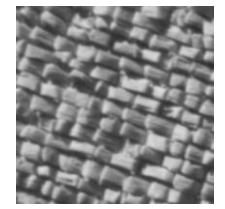

$20^{\circ}$

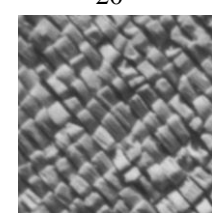

$50^{\circ}$

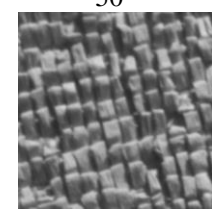

$80^{\circ}$
Fig.3. Sample images of Brodatz D84 texture class with orientation angles $0^{\circ}$ through $90^{\circ}$ in steps of $10^{\circ}$. 


\section{2) Mondial Marmi}

The dataset has twelve texture classes of granite tile images popularly used for color and texture analysis, namely, Rosa Porrio B, Rosa Porrio A, Rosa Beta, Giallo Veneziano, Criallo Santa Cecilia, Giallo Ornamentale, Giallo Napoletano, Bianco Sardo, Bianco Cristal, Azul Platino, Azul Capixaba, Acquamarina. The dataset has four images, each rotated by nine different angles $\left(0^{\circ}, 5^{\circ}\right.$, $\left.10^{\circ}, 15^{\circ}, 30^{\circ}, 45^{\circ}, 60^{\circ}, 75^{\circ}, 90^{\circ}\right)$ per class. Each image is subdivided into 4 non-overlapping subimages of size 272 x 272 [30]. The sample images of the texture class BiancoCristal_A for different rotation angles considered in the experimentations are shown in Fig. 4.

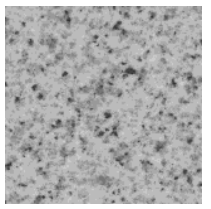

$0^{\circ}$

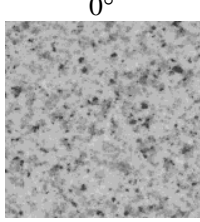

$15^{\circ}$

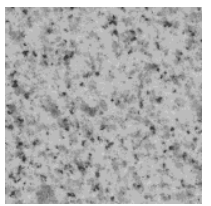

$60^{\circ}$

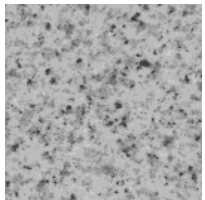

$5^{\circ}$

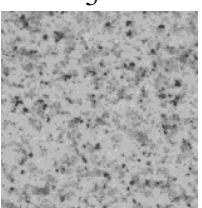

$30^{\circ}$

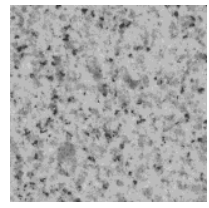

$75^{\circ}$

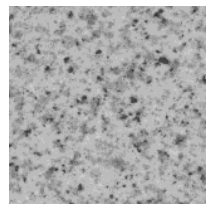

$10^{\circ}$

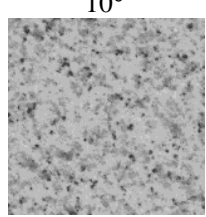

$45^{\circ}$

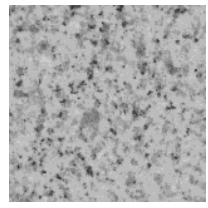

$90^{\circ}$
Fig.4. Sample images of Mondial Marmi BiancoCristal_A texture class with orientation angles $0^{\circ}$ through $90^{\circ}$.

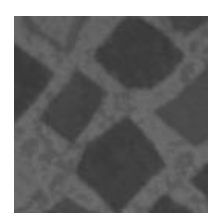

$0^{\circ}$

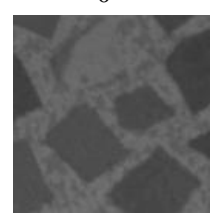

$15^{\circ}$

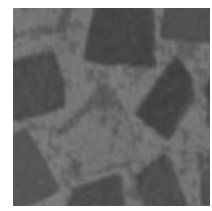

$60^{\circ}$

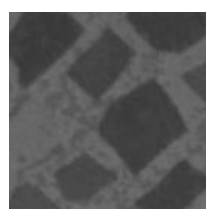

$5^{\circ}$

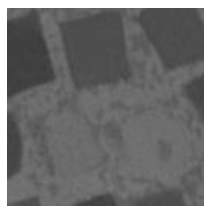

$30^{\circ}$

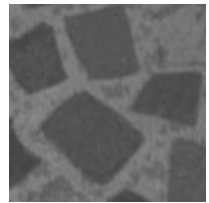

$75^{\circ}$

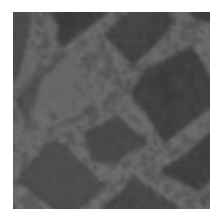

$10^{\circ}$

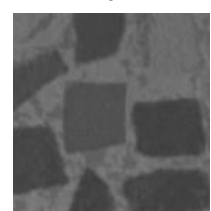

$45^{\circ}$

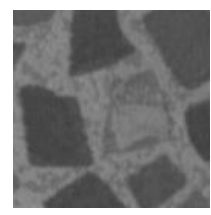

$90^{\circ}$
Fig.5. Sample images of Outex plastic 001 texture class with orientation angles $0^{\circ}$ through $90^{\circ}$.

\section{3) Outex}

Outex is a widely used dataset for texture analysis. The images present in Outex_0045 are of 45 different classes. The original images are subdivided into 20 nonoverlapping subimages of resolutions $128 \times 128$. These images are rotated by nine different angles $\left(0^{\circ}, 5^{\circ}, 10^{\circ}\right.$, $\left.15^{\circ}, 30^{\circ}, 45^{\circ}, 60^{\circ}, 75^{\circ}, 90^{\circ}\right)$ [31]. The sample images of the texture class plastic001 for different rotation angles are shown in Fig. 5.

\section{4) Vectorial}

The Vectorial dataset contains 20 texture classes of artificial images. The texture classes are Abstract $\{1,2,3$, $4\}$, circles $\{1,2\}$, flowers $\{1,2,3\}$, leaves $\{1,2\}$, squares $\{1,2\}$, wallpaper $\{1,2,3,4,5,6,7\}$. The original images are subdivided into $4 \mathrm{x} 4$ non-overlapping samples of size $225 \times 225$ Each image is rotated by $10^{\circ}$ ranging from $0^{\circ}$ to $90^{\circ}$ [32]. The sample images of the texture class Wallpaper_1 for different rotation angles are shown in Fig. 6.

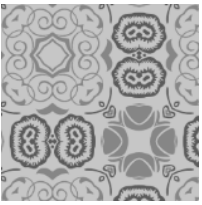

$0^{\circ}$

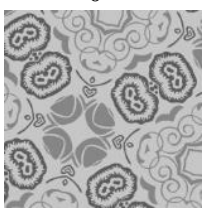

$30^{\circ}$

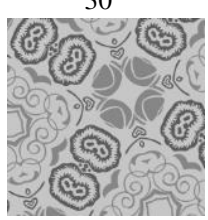

$60^{\circ}$

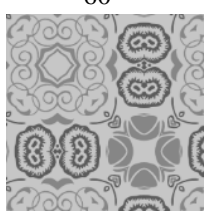

$90^{\circ}$

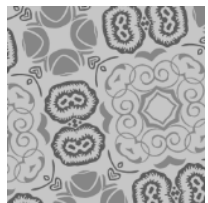

$10^{\circ}$

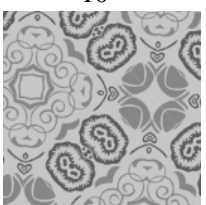

$40^{\circ}$

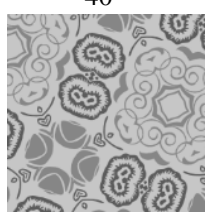

$70^{\circ}$

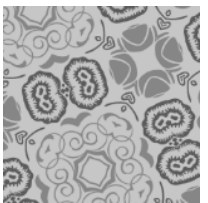

$20^{\circ}$

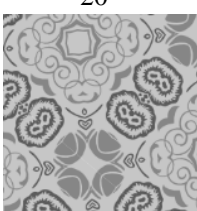

$50^{\circ}$

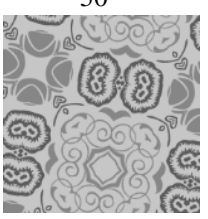

$80^{\circ}$
Fig.6. Sample images of Vectorial Wallpaper_1 texture class with orientation angles $0^{\circ}$ through $90^{\circ}$ in steps of $10^{\circ}$.

\section{B. Results and Discussion}

Three different types of experimentation have been conducted, i.e. Rotation invariant, Scale invariant and Rotation-and-Scale invariant classification, to show the effectiveness of the proposed method.

\section{1) Rotation invariant classification}

In all the experiments for rotation invariance, the unrotated image (i.e. image at $0^{\circ}$ ) is used as the training 
sample and the images with different orientation angles are considered as the test samples. The experimental results are presented in Tables 1 and 2. Table 1 shows the classification results obtained using the proposed method and other state-of-the-art methods on Brodatz dataset. The feature vector consists of the normalized cumulative histogram bin values extracted from the Galois Field operated images. The texture image is rotated in steps of $10^{\circ}$ upto $90^{\circ}$. Hence the number of orientations used for rotation invariant classification is 10 . For the rotation invariant classification, a total of 208 x 10 images are used from the Brodatz dataset. The results obtained using the proposed method is compared with the original Local Binary Pattern method for rotation invariance (RI LBP) and Log-Polar transform method. The K-Nearest
Neighbour classifier is used for classification, where the values of $\mathrm{K}=1,3$ and 5 are considered for the experimentation. As observed from Table 1, the Galois Field operator method classified the images with $93.17 \%$ accuracy as against the classification rate of $86.48 \%$ for RILBP and $91.25 \%$ for Log Polar transform method for $\mathrm{K}=1$. The classification results of the proposed method are also better when compared to other two methods for $\mathrm{K}=3$ and $\mathrm{K}=5$. The similar experiments are also performed on other three different datasets for $K=1$. The average classification rate over all the angles on different datasets is shown in Table 2. From Tables 1 and 2, it is observed that Galois Field operator method outperforms RILBP and Log Polar transform method.

Table 1. Comparison of average classification rate over varying rotated angles on Brodatz dataset obtained using proposed method with RILBP and Log-Polar transform for $\mathrm{K}=1,3$ and 5.

\begin{tabular}{|c|c|c|c|c|c|c|c|c|c|}
\hline \multirow{2}{*}{ Testing Angles } & \multicolumn{3}{|c|}{ RILBP [10](\%) } & \multicolumn{3}{c|}{ Log-Polar Transform [23] (\%) } & \multicolumn{3}{c|}{ GF operator (proposed method) (\%) } \\
\cline { 2 - 12 } & $\mathrm{K}=1$ & $\mathrm{~K}=3$ & $\mathrm{~K}=5$ & $\mathrm{~K}=1$ & $\mathrm{~K}=3$ & $\mathrm{~K}=5$ & $\mathrm{~K}=1$ & $\mathrm{~K}=3$ & $\mathrm{~K}=5$ \\
\hline 0 & 100 & 98.56 & 97.60 & 100 & 93.75 & 89.42 & 100 & 92.31 & 88.46 \\
\hline 10 & 87.98 & 87.50 & 83.66 & 89.42 & 85.58 & 80.77 & 91.83 & 92.31 & 88.94 \\
\hline 20 & 80.77 & 80.77 & 79.81 & 93.75 & 87.50 & 82.70 & 95.67 & 93.75 & 91.35 \\
\hline 30 & 82.69 & 81.25 & 80.77 & 90.87 & 88.94 & 86.53 & 95.19 & 92.31 & 90.39 \\
\hline 40 & 83.17 & 83.65 & 82.22 & 91.35 & 86.06 & 83.65 & 91.35 & 91.35 & 87.02 \\
\hline 50 & 82.21 & 81.25 & 79.33 & 89.90 & 85.10 & 82.69 & 89.90 & 87.50 & 87.50 \\
\hline 60 & 73.56 & 72.12 & 74.52 & 87.02 & 85.10 & 82.69 & 88.46 & 87.50 & 86.06 \\
\hline 70 & 80.29 & 79.81 & 79.81 & 89.42 & 89.91 & 83.17 & 91.83 & 92.79 & 87.98 \\
\hline 80 & 87.02 & 86.06 & 84.62 & 90.87 & 85.58 & 82.69 & 94.71 & 90.87 & 88.94 \\
\hline 90 & 97.12 & 96.16 & 97.12 & 89.90 & 88.46 & 82.69 & 92.79 & 92.79 & 90.38 \\
\hline Mean & $\mathbf{8 5 . 4 8}$ & $\mathbf{8 4 . 7 1}$ & $\mathbf{8 3 . 9 4}$ & $\mathbf{9 1 . 2 5}$ & $\mathbf{8 7 . 6 0}$ & $\mathbf{8 3 . 7 1}$ & $\mathbf{9 3 . 1 7}$ & $\mathbf{9 1 . 3 5}$ & $\mathbf{8 8 . 7 1}$ \\
\hline
\end{tabular}

Table 2. Average classifications rates $(\%)$ over all the angles on different datasets for $\mathrm{K}=1$ obtained by proposed GF operator, RILBP and Log-Polar transform methods.

\begin{tabular}{|c|c|c|c|}
\hline Datasets & RI LBP [10](\%) & Log-Polar Transform [23](\%) & GF operator (proposed method)(\%) \\
\hline Brodatz & 85.48 & 91.25 & 93.17 \\
\hline Mondial Marmi & 84.73 & 90.74 & 93.11 \\
\hline Outex & 77.74 & 75.99 & 80.05 \\
\hline Vectorial & 74.41 & 96.34 & 96.25 \\
\hline
\end{tabular}

Table 3. Classifications rate (\%) over different scales 1.2 to 3.0 in steps of 0.2 on four different datasets obtained by the proposed method for $\mathrm{K}=1$.

\begin{tabular}{|c|c|c|c|c|}
\hline Scale & Brodatz & Mondial Marmi & Outex & Vectorial \\
\hline 1.0 & 67.3077 & 63.0208 & 30.5556 & 91.5625 \\
\hline 1.2 & 82.6923 & 73.4375 & 59.1111 & 95 \\
\hline 1.4 & 89.9038 & 82.2917 & 75.8889 & 95.9375 \\
\hline 1.6 & 91.3462 & 87.5000 & 86.2222 & 99.0625 \\
\hline 1.8 & 97.5692 & 95.8333 & 96.3333 & 100 \\
\hline 2.0 & 100 & 100 & 100 & 100 \\
\hline 2.2 & 99.0385 & 96.3542 & 96.5556 & 100 \\
\hline 2.4 & 95.1923 & 86.9792 & 87.1111 & 99.3750 \\
\hline 2.6 & 93.7500 & 80.7392 & 80.4444 & 99.3750 \\
\hline 2.8 & 90.3846 & 78.1250 & 75.2222 & 97.1875 \\
\hline 3.0 & 88.4615 & 75.5208 & 69.4444 & 95 \\
\hline Average & $\mathbf{9 0 . 5 1 5 7}$ & $\mathbf{8 3 . 6 1 7 4}$ & $\mathbf{7 7 . 8 9 8 9}$ & $\mathbf{9 7 . 5}$ \\
\hline
\end{tabular}


Table 4. Comparison of average classification rates (\%) over all the scales considered on different datasets obtained by using proposed method with RILBP and Log-Polar transform for $\mathrm{K}=1$.

\begin{tabular}{|c|c|c|c|}
\hline Datasets & RI LBP [10](\%) & Log-Polar Transform [23](\%) & GF operator (proposed method)(\%) \\
\hline Brodatz & 39.2482 & 88.7674 & 80.5157 \\
\hline Mondial Marmi & 33.6647 & 81.2481 & 77.8989 \\
\hline Outex & 19.4949 & 77.2222 & 97.5 \\
\hline Vectorial & 54.5736 & 97.4715 & \multirow{2}{*}{} \\
\hline
\end{tabular}

Table 5. Classification rate $(\%)$ over all the orientation angles and scales on four different datasets for $\mathrm{K}=1$.

\begin{tabular}{|c|c|c|c|}
\hline Datasets & RILBP [10](\%) & $\begin{array}{c}\text { Log-Polar Transform } \\
{[\mathbf{2 3}](\mathbf{\%})}\end{array}$ & GF operator (proposed method)(\%) \\
\hline Brodatz & 37.8702 & 79.7404 & 82.3846 \\
\hline Mondial Marmi & 33.5938 & 74.1956 & 77.3611 \\
\hline Outex & 16.4926 & 60.1617 & 63.2012 \\
\hline Vectorial & 46.1125 & 90.0812 & 89.0563 \\
\hline
\end{tabular}

\section{2) Scale invariant classification}

For scale invariance, the texture images in the database are scaled by scaling factor of 1.0 to 3.0 with step 0.2 . The sample images of texture classes of Brodatz, Mondial Marmi, Outex and Vectorial datasets for scales 1.2 to 3.0 in steps of 0.2 are shown in Fig 7, Fig 8, Fig 9 and Fig 10 respectively. The images of scale factor 2.0 are used as the training samples and the remaining images of different scales are used as testing samples. The Galois Field operator method is applied on each scaled image. For scale invariant classification, $208 \times 10$ images from the Brodatz dataset, $192 \times 10$ images from Mondial Marmi, 900 x 10 images from Outex and 320 x 10 images from Vectorial dataset are considered. Table 3 shows the classification rates obtained by proposed method over the different scales for the four different datasets. The comparison of average percentage of classification over all the scales on four different datasets obtained by using the proposed method over RILBP and Log-Polar transform is presented in Table 4. From the Table 4, it is seen that the proposed method outperforms the other two methods.

\section{3) Rotation-and-Scale invariant classification}

For the experimentation, the datasets are created by first rotating the images and then scaling the rotated images to different sizes. The images are cropped from the center to maintain the original size of the image. The unrotated $\left(\right.$ at $0^{\circ}$ ) and scaled images with scale factor 2 are chosen as the training samples and the remaining rotated and scaled images are the testing samples for the evaluation of performance of the proposed method. Table 5 shows the classification performance obtained by the proposed method on rotated and scaled images for four different datasets. From Table 5, it is observed that the

proposed method has better classification rates when compared to other two methods.

Over all, it is also observed that, only in case of Vectorial dataset, the performance of proposed GF operator is comparable to Log-Polar transform. In case of other three datasets, proposed GF operator outperforms RILBP and Log-Polar transform.
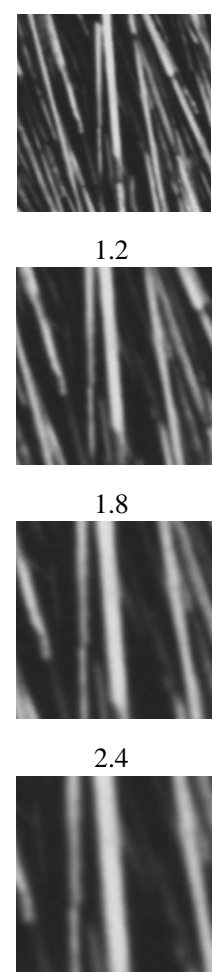

3.0

Fig.7. Sample images of Brodatz D15 texture class for all scale factors 1.2 to 3.0 in steps of 0.2
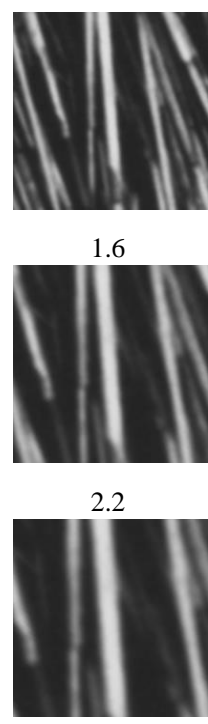

2.8 


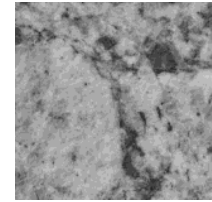

1.2

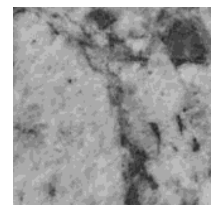

1.8

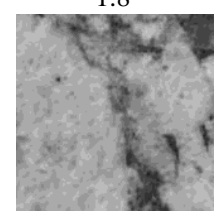

2.4

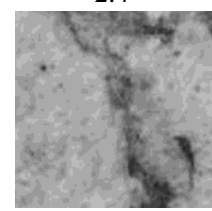

3.0

Fig.8. Sample images of Mondial Marmi GialloNapoletano_A_1 texture class for all scale factors 1.2 to 3.0 in steps of 0.2

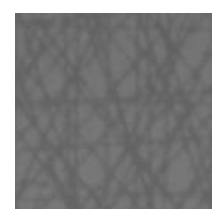

12

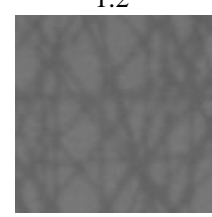

1.8

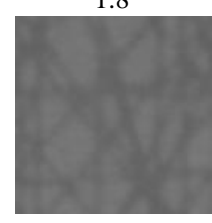

2.4

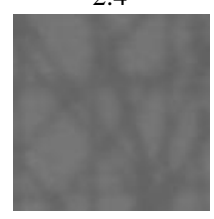

3.0

Fig.9. Sample images of Outex plastic038 texture class for all scale factors 1.2 to 3.0 in steps of 0.2
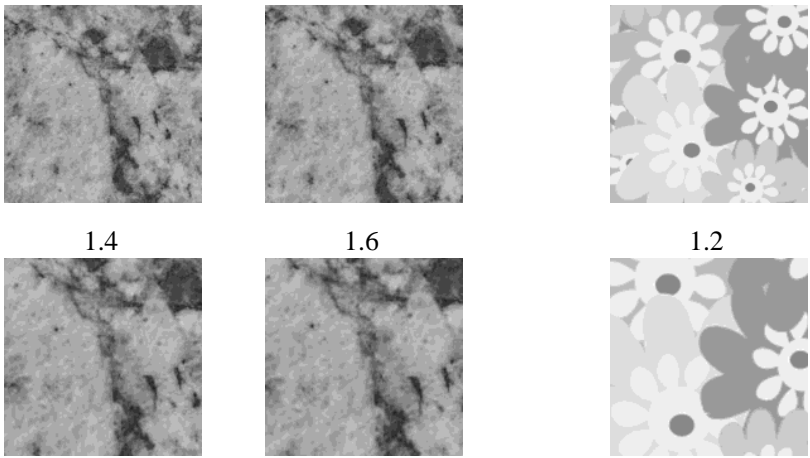

2.0

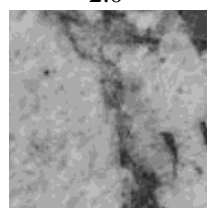

2.6
1.6
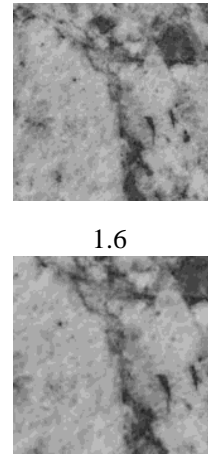

2.2

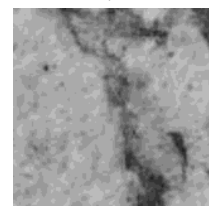

2.8

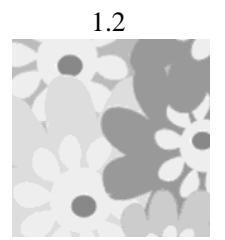

1.8

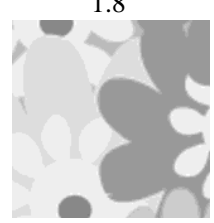

2.4

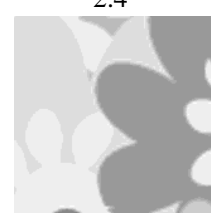

3.0

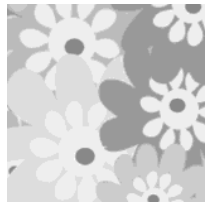

1.4

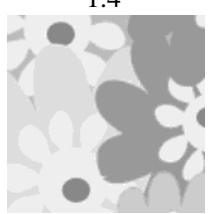

2.0

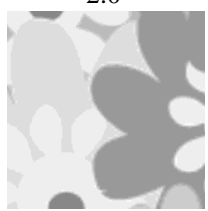

2.6

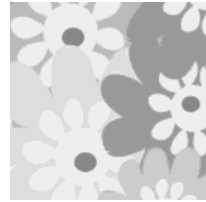

1.6

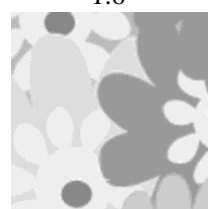

2.2

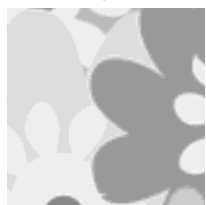

2.8
Fig.10. Sample images of Vectorial Flowers_1 texture class for all scale factors 1.2 to 3.0 in steps of 0.2

\section{CONCLUSION}

In this paper, a novel approach using Galois Field addition operator is proposed for rotation and scale invariant texture classification. The commutative and associative properties of Galois Field addition are helpful in achieving the rotation and scale invariance of texture classification. The effectiveness of the proposed GF approach has been demonstrated experimentally using four benchmark texture datasets, namely, Brodatz [29], Mondial Marmi [30], Outex [31] and Vectorial [32] of planar images. The results obtained by using the proposed GF approach are compared with that of RILBP and LogPolar transform methods. It is observed that the proposed GF approach is more effective as compared to other two methods in achieving rotation and scale invariance.

\section{REFERENCES}

[1] S. Purohit and S. R. Gandhi, "Application of Sparse Coded SIFT Features for Classification of Plant Images". International Journal of Image, Graphics and Signal Processing, vol 9(10), pp. 50-59, 2017. "doi: 10.5815/ijigsp.2017.10.06 “

[2] K.S. Reddy, V.V. Kumar and B.E. Reddy, "Face recognition based on texture features using local ternary patterns", International Journal of Image, Graphics and Signal Processing, vol 7(10),pp. 37-46, 2015. “doi: 10.5815/ijigsp.2015.10.05“ 
[3] T.P. Nguyen, N.S. Vu, and A. Manzanera, "Statistical binary patterns for Rotational Invariant Texture Classification", Neurocomputing, vol. 173, pp. 1565-1577, 2016."doi:10.1016/j.neucom.2015.09.029"

[4] R.K. Goyal, W.L. Goh, D.P. Mital and K.L. Chan, "Scale and Rotation Invariant Texture Analysis based on Structural property", in Industrial Electronics, Control, and Instrumentation, 1995., Proceedings of the 1995 IEEE IECON 21st International Conference on, Vol. 2, pp. 1290-1294, IEEE, November, 1995. "doi: 10.1109/IECON.1995.483983"

[5] Y.Q. Chen, M.S. Nixon and D.W. Thomas, "Statistical Geometrical Features for Texture Classification", Pattern Recognition, vol. 28(4), pp. 537-552, 1995. “doi: 10.1016/0031-3203(94)00116-4",

[6] C. Dharmagunawardhana, S. Mahmoodi, M. Bennett and M. Niranjan, "Rotation Invariant Texture Descriptors based on Gaussian Markov random fields for Classification", Pattern Recognition Letters, vol. 69, pp. 15-21, 2016."doi: 10.1016/j.patrec.2015.10.006"

[7] F. Bianconi and A. Fernández, "Rotation Invariant Cooccurrence features based on digital circles and Discrete Fourier Transform", Pattern Recognition Letters, vol. 48, pp. 34-41, 2014."doi: 10.1016/j.patrec.2014.04.006"

[8] P. Simon and V. Uma, "Review of Texture Descriptors for Texture Classification", In Data Engineering and Intelligent Computing pp. 159-176, Springer, Singapore, 2018. "doi: 10.1007/978-981-10-3223-3 15"

[9] R.L. Kashyap and A. Khotanzad, "A model-based method for Rotation Invariant Texture Classification", IEEE Transactions on Pattern Analysis and Machine Intelligence, vol. 4, pp. 472-481, 1986."doi: 10.1109/TPAMI.1986.4767811"

[10] T. Ojala, M. Pietikainen and T. Maenpaa, "Multiresolution Gray-scale and Rotation Invariant Texture Classification with Local Binary Patterns", IEEE Transactions on pattern analysis and machine intelligence, vol. 24(7), pp. 971-987, 2002. “doi: 10.1109/TPAMI.2002.1017623"

[11] R. Porter and N. Canagarajah, "Robust Rotation-Invariant Texture Classification: wavelet, Gabor filter and GMRF based schemes", IEEE Proceedings-Vision, Image and Signal Processing, vol. 144(3), pp. 180-188, 1997. “doi: 10.1049/ip-vis:19971182”

[12] S. Arivazhagan, L. Ganesan and S.P. Priyal, "Texture classification using Gabor wavelets based Rotation Invariant features", Pattern Recognition Letters vol. 27(16), pp. 1976-1982, 2006."doi: 10.1016/j.patrec.2006.05.008"

[13] S. Bharkad and M. Kokare, "Rotation-invariant fingerprint matching using Radon and DCT", Sadhana, vol. 42(12), pp. 2025-2039, 2017."'doi: 10.1007/s12046017-0752-3"

[14] T. Song, H. Li, F. Meng, Q. Wu, and J. Cai, "Letrist: Locally Encoded Transform feature histogram for Rotation-Invariant Texture Classification", IEEE Transactions on Circuits and Systems for Video Technology, 2017. "doi: 10.1109/TCSVT.2017.2671899"

[15] S.K. Roy, N. Bhattacharya, B. Chanda, B.B. Chaudhuri, and D.K. Ghosh, "FWLBP: A Scale Invariant Descriptor for Texture Classification", arXiv preprint arXiv:1801.03228, 2018.
[16] Y. Xu, H. Ji, and C. Fermüller, "Viewpoint invariant texture description using Fractal Analysis", International Journal of Computer Vision, vol. 83(1), pp. 85-100, 2009. "doi: 10.1007/s11263-009-0220-6"

[17] L. Liu, P. Fieguth, G. Kuang, and H. Zha, "Sorted random projections for robust Texture Classification", In Computer Vision (ICCV), 2011 IEEE International Conference on, pp. 391-398, IEEE, November, 2011."doi: 10.1109/ICCV.2011.6126267"

[18] J. Zhang, M. Marszałek, S. Lazebnik, and C. Schmid, "Local features and kernels for Classification of Texture and Object Categories: A comprehensive study", International Journal of Computer Vision, vol. 73(2), pp. 213-238, 2007. "doi: 10.1007/s11263-006-9794-4"

[19] M. Crosier, and L.D. Griffin, "Using basic image features for Texture Classification", International Journal of Computer Vision, vol. 88(3), pp. 447-460, 2010. “doi: 10.1007/s11263-009-0315-0"

[20] J. Zhang, and T. Tan, "Affine invariant Classification and Retrieval of Texture Images", Pattern Recognition, vol. 36(3), pp. 657-664, 2003. “doi: 10.1016/S00313203(02)00099-7"

[21] Y. Quan, Y. Xu, and Y. Sun, "A distinct and compact texture descriptor", Image and Vision Computing, vol. 32(4), pp. 250-259, $2014 . \quad$ "doi: 10.1016/j.imavis.2014.02.004"

[22] J. Mao, and A.K. Jain, "Texture classification and segmentation using Multiresolution Simultaneous Autoregressive Models", Pattern Recognition, vol. 25(2), pp. 173-188, 1992. "doi: 10.1016/0031-3203(92)90099-5"

[23] C.M. Pun, and M.C. Lee, "Log-polar Wavelet Energy Signatures for Rotation and Scale Invariant Texture Classification", IEEE transactions on pattern analysis and machine intelligence, vol. 25(5), pp. 590-603, 2003. "doi: 10.1109/TPAMI.2003.1195993"

[24] J. Han, and K.K Ma, "Rotation-invariant and Scaleinvariant Gabor features for texture image retrieval", Image and vision computing, vol. 25(9), pp. 1474-1481, 2007. "doi: 10.1016/j.imavis.2006.12.015"

[25] J. Zhang, and T. Tan, "Brief review of invariant texture analysis methods", Pattern Recognition, vol. 35(3), pp. 735-747, 2002.

[26] I.S. Reed, T.K. Truong, Y.S. Kwoh, and E.L. Hall, "Image Processing by Transforms over a Finite Field", IEEE Transactions on Computers, vol. (9), pp. 874-881, 1977. "doi: 10.1109/TC.1977.1674935"

[27] S. Shivashankar, M. Kudari and P.S. Hiremath,” Texture Representation Using Galois Field for Rotation Invariant Classification". In Signal-Image Technology \& InternetBased Systems (SITIS), 2017 13th International Conference on. IEEE. pp. 237-240, Dec 2017. "doi: 10.1109/SITIS.2017.48"

[28] R.O. Duda, P.E. Hart, and D.G. Stork, Pattern Classification (Vol. 2). New York: Wiley, 1973.

[29] P. Brodatz, Textures: a photographic album for artists and designers, Dover Publications, New York, USA, 1966.

[30] MondialMarmi database, Available online at http://dismac.dii.unipg.it/mm/ver_1_1/index.html

[31] OuTeX, OuTeX database, Available online at http://www.outex.oulu.fi/, 2002.

[32] All-free-download, Available online at http:// all-freedownload.com, 2012. 


\section{Authors' Profiles}

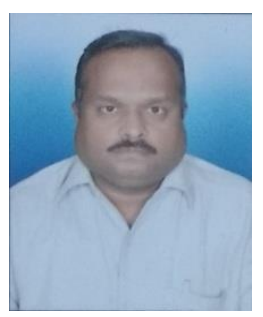

Dr. Shivashankar S. has completed Master of Computer Application (M.C.A.) degree in 2000 and obtained Ph.D. in Computer Science in 2007 from Gulbarga University, Gulbarga, Karnataka, India. Presently he is working as Associate Professor in the Department of Computer Science, Karnatak University, Dharwad Karnataka, India. His research area of interest is Digital Image Processing, Pattern Recognition, Computer Vision and Machine Learning and Data Mining. He has published more than 25 research papers in various peer reviewed Inter-national Journals and Conferences.

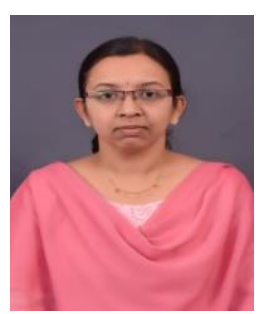

Ms. Medha Kudari has completed M.Sc in Computer Science in 2005 from Mangalore University and is presently pursuing Ph.D under the guidance of Dr. Shivashankar S. at Karnatak University, Dharwad. Her areas of research are Digital Image Processing and Pattern Recognition.

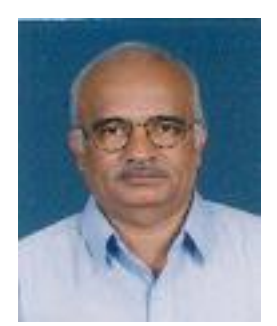

Dr. Prakash S. Hiremath has obtained M.Sc. degree in 1973 and Ph.D. degree in 1978 in Applied Mathematics from Karnatak University, Dharwad. He had been in the Faculty of Mathematics and Computer Science of various Institutions in India, namely, National Institute of Technology, Surathkal (1977-79), Coimbatore Institute of Technology, Coimbatore (1979-80), National Institute of Technology, Tiruchinapalli (1980-86), Karnatak University, Dharwad (19861993). From 1993-2014 he worked as a Professor in Department of Computer Science, Gulbarga University, Gulbarga. Presently, working as Professor, Department of Computer Science (MCA), KLE Technological University, Hubballi, Karnataka, India. His research areas of interest are Computational Fluid Dynamics, Optimization Techniques, Image Processing and Pattern Recognition, and Computer Networks. He has published more than 210 research papers in peer reviewed International Journals.

How to cite this paper: Shivashankar S., Medha Kudari, Prakash S. Hiremath, " Galois Field-based Approach for Rotation and Scale Invariant Texture Classification", International Journal of Image, Graphics and Signal Processing(IJIGSP), Vol.10, No.9, pp. 56-64, 2018.DOI: 10.5815/ijigsp.2018.09.07 